\title{
Responsiveness and minimum important change of the Oswestry Disability Index in Italian subjects with symptomatic lumbar spondylolisthesis
}

\author{
Carla Vanti $^{1,5} \cdot$ Silvano Ferrari $^{1} \cdot$ Jorge Hugo Villafañe $^{2} \cdot$ Pedro Berjano $^{3} \cdot$ \\ Marco Monticone ${ }^{4}$
}

Received: 21 February 2016/ Accepted: 23 January 2017/Published online: 16 February 2017

(c) The Author(s) 2017. This article is published with open access at Springerlink.com

\begin{abstract}
Background This study aims to investigate the responsiveness and the minimum important change of the Italian version of the Oswestry Disability Index (ODI-I) in subjects with symptomatic specific low back pain associated with lumbar spondylolisthesis (SPL).

Materials and methods One hundred and fifty-one patients with symptomatic SPL completed the ODI-I, a 0-100 numerical rating scale (NRS), and performed the prone and supine bridge tests. The global perception of effectiveness was measured with a 7-point Likert scale. Responsiveness was assessed by distribution methods (minimum detectable change [MDC], effect size [ES], standardized response mean $[\mathrm{SRM}]$ ) and anchor-based methods (ROC curves).
\end{abstract}

Carla Vanti

carla.vanti@unibo.it

Silvano Ferrari

silvano.ferrari@fastwebnet.it

Jorge Hugo Villafañe

mail@villafane.it

Pedro Berjano

pberjano@gmail.com

Marco Monticone

marco.monticone@unica.it

1 Department of Molecular Medicine, University of Padova, Padua, Italy

2 IRCCS Don Gnocchi Foundation, Milan, Italy

3 IRCCS Istituto Ortopedico Galeazzi, Milan, Italy

4 Department of Public Health, Clinic and Molecular Medicine, University of Cagliari, Cagliari, Italy

5 Via Tosarelli 144, Castenaso, 40055 Bologna, Italy
Results The MDC was 4.23, the ES was 0.95 and the SRM was 1.25. ROC analysis revealed an area under the curve of 0.76 indicating moderate discriminating capacity. The best cut-off point for the dichotomous outcome was 7.5 (sensitivity $90.3 \%$, specificity $56.7 \%$ ). .

Conclusions The ODI-I proved to be responsive in detecting changes after conservative treatment in subjects with lumbar SPL.

Level of evidence II.

Keywords Spondylolisthesis - Low back pain . Responsiveness · Oswestry Disability Index · Outcome measures

\section{Introduction}

The ability of a scale to be sensitive to change (responsiveness) is important not only in the clinical setting, but also for research, allowing power calculations, sample size estimates and cost evaluations [1]. When a scale is applicable on a wide range of clinical conditions, it is necessary to investigate whether the baseline scores and the change in scores are similar on the various categories of subjects to which the scale is administered or, conversely, whether the different diagnostic subgroups show dissimilar results [2].

The Oswestry Disability Index (ODI) is one of the most widely used questionnaires measuring low back pain (LBP)-related disability [3]. It is a self-administered 10-item questionnaire, composed by one section rating the intensity of pain and nine others describing the disabling effect of LBP on daily activities. The score for each item ranges from $0-5$, and the sum of the ten scores is expressed as a percentage of the maximum score, ranging from 0 (no disability) to 100 (maximum disability). The 
values proposed for the minimum important change of ODI are a reduction of 10 points, or a decrease of $30 \%$ compared to the initial value [4].

The ODI has been translated and culturally adapted into several languages, including Italian [5], and its responsiveness was investigated in Italian subjects complaining of subacute and chronic non-specific LBP. The minimal detectable change (MDC) for the ODI was 13.67, the effect size (ES) was 0.53 , and the standardized response mean (SRM) was 0.80 . The best cut-off point for the dichotomous outcome was 9.5 (sensitivity $76 \%$, specificity 63\%). ROC analysis revealed an area under the curve of 0.71 . ODI moderately correlated with the numerical rating scale (NRS). These results were consistent with other published studies on non-specific LBP [6].

International literature investigated the psychometric properties of the ODI in different LBP subgroups, including spondylolisthesis (SPL). The weighted main ODI score in SPL was calculated on 120 subjects (pooled data from different studies) as 26.63, and the weighted mean difference as 14.4 [2]. Nevertheless, these pooled data came from studies conducted in very different settings-four studies on surgically treated patients [7-10], and one study involving 44 subjects on conservatively treated patients [11].

To the best of our knowledge, there is only one other study on the responsiveness of the ODI in a conservative setting for SPL-a non-randomized trial of 20 patients in which the main ODI score in SPL was 30.35 and the mean difference was 10.20 [12].

No study has been conducted on the Italian version of the ODI (ODI-I) in clinical conditions different from nonspecific LBP. The objective of this study is to examine the responsiveness and the minimum important change of the ODI-I in Italian subjects with symptomatic specific LBP associated with lumbar SPL undergoing a physical therapy program.

\section{Materials and methods}

\section{Design}

A prospective cohort observational study was conducted. The present paper was prepared according to the editorial form of medical publishing and STROBE publishing rules [13].

\section{Participants}

A total of 151 subjects with symptomatic lumbar SPL were diagnosed according to the gold criteria [14], by the referring orthopedic doctors or spinal surgeons. Before starting the conservative treatment, the patients were informed about the different therapeutic options by their specialist, and a shared decision was reached. All patients complained of LBP, namely pain, muscle tension, or stiffness localized below the costal margin and above the inferior gluteal folds, with or without sciatica.

All patients were clinically stable and they all underwent a physical examination by two physical therapists with expertise in orthopedic manual therapy. The examiners verified the presence of Waddell's signs, which are suggested by Italian LBP Guidelines to exclude the presence of non-organic pain, due to a major psychological or social involvement [15].

The inclusion criteria were a diagnosis of symptomatic SPL, aged $>18$ years, a diagnosis of SPL confirmed by X-ray, CT or MRI, level L4/L5 or L5/S1, isthmic or degenerative types [15], and the ability to speak and write in Italian. Subjects who had undergone previous lumbar surgery, who were affected by systemic diseases (e.g., inflammatory or infectious pathologies, cancer, etc.), spinal specific pathologies (e.g., spinal stenosis, inflammatory spinal diseases such as ankylosing spondylitis, discitis, and arachnoiditis), neuromuscular disorders, cognitive deficits, or who did not sign the informed consent were excluded.

\section{Outcome measures}

Two physical therapists with expertise in orthopedic manual therapy collected the measurements and administered the treatments. The NRS [16] and the ODI-I [5] were administered to measure pain and disability, respectively. Subjects also completed a global perception effect (GPE) questionnaire. This questionnaire is a 7-point Likert-type scale comprising only one question to evaluate the subject's self-reported improvement or deterioration after the intervention. Two clinical tests commonly used to detect muscle endurance were performed - the prone bridge test (PBT) and the supine bridge test (SBT) [17].

\section{Procedures}

The patients signed the informed consent, provided demographic and clinical data, and completed the ODI-I and the NRS. A specific schedule was prepared to collect main comorbidities. All the forms were placed in a closed envelope. The physical therapists then asked the subjects to perform the bridge tests, and the results were recorded on a separate form.

The SBT was performed in the supine position, asking the subject to raise his/her pelvis from the table so that his/ her shoulders, hips, and knees were maintained in a straight line. The PBT was performed in the prone position, asking the subject to raise his/her pelvis from the table so that only 
his/her forearms and his/her toes were in contact with the table. These positions were sustained until fatigue or pain prevented the maintenance of the test position and the physical therapists recorded the holding time in seconds.

Patients attended physical therapy treatment for a number of sessions and over a period depending on the individual patient's needs. Each session lasted $1 \mathrm{~h}$, and included supervised exercises and home exercises aiming to improve lumbar stability, according to the therapeutic program proposed by Richardson et al. [18].

Progressively, the exercises involved all lumbar muscles, increasing range of movement, load and speed and advancing towards more complex movement patterns, and maintaining muscle stabilization. This program was found to be effective in a previous study [19].

A functional and graded approach was also performed to increase activity level and improve strength, endurance, range of motion, balance, and coordination [20].

Immediately at the end of the treatment, patients completed the ODI-I, NRS and GPE questionnaires, which were placed in a closed envelope, and physical therapists recorded the results of the bridge tests. Post-treatment testing was performed by the same assessor who carried out the pre-treatment measurements. The administrative staff created an electronic database with the collected data.

\section{Statistical analysis}

Data were analyzed using SPSS version 21.0 (SPSS Inc, Chicago, IL, USA).

Responsiveness was assessed by means of distribution methods-MDC, ES, SRM and anchor-based methods (ROC curves) [20]. Spearman's rank correlation coefficient $\left(R_{\mathrm{s}}\right)$ was used to evaluate the relationship between the ODI and other parameters evaluated. The $R_{\mathrm{s}}$ values were interpreted according to Domholdt's recommendations.

Statistical analysis was conducted at a $95 \%$ confidence level, and $P<0.05$ was considered statistically significant.

\section{Results}

One hundred and sixty-eight consecutive subjects with symptomatic SPL were screened for eligibility criteria. One hundred and fifty-one satisfied all eligibility criteria and agreed to participate (Fig. 1). The mean age was $45 \pm 15$ years, with $62.9 \%$ women. The mean number of sessions was $8 \pm 2$ and the mean duration of the treatment was $2 \pm 1$ months. The characteristics of the sample are shown in Table 1. All subjects attended the treatment sessions and completed the follow-up.
168 subjects with symptomatic lumbar

Spondylolisthesis were assessed

15 subjects were excluded:

- 5 had inflammatory spinal disease

- 3 had peripheral neuropathy

- 5 had previous spinal surgery

- 2 had lumbar stenosis

153 subjects with symptomatic lumbar

Spondylolisthesis remained

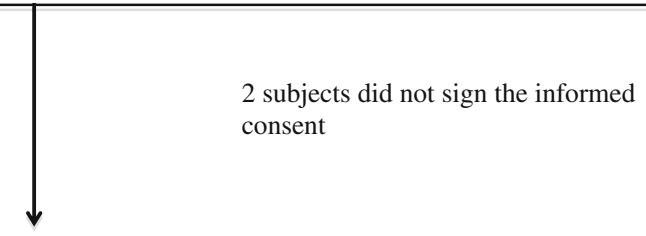

151 subjects participated to the study

Fig. 1 Flow chart

The main ODI score at the beginning of the treatment was $22.8 \pm 12.9$ and the main post-treatment change was $-10.7 \pm 0.9$. All other outcome measures (NRS, PBT, and SBT) showed statistically significant improvements after the period of treatment (Table 2).

Sixty-eight $(45 \%)$ of the subjects reported 'completely better', $52(34.4 \%)$ reported 'much better' only, and the remaining $31(20.6 \%)$ reported both 'little better' and 'about the same'.

The MDC was 4.23 , the ES was 0.95 and the SRM was 1.25; ROC analysis revealed an area under the curve of 0.76 indicating moderate discriminating capacity. The best cut-off point for the dichotomous outcome was 7.5 (sensitivity $90.3 \%$, specificity $56.7 \%$ ) (Table 3 ).

Spearman's rank correlation coefficients showed a moderately, significant and negative relationship between the ODI-I and PBT and SBT $\left(R_{\mathrm{s}}=-0.5\right.$ and -0.48 , respectively, both $P<0.001)$ and a good, significant and direct relationship between the ODI-I and the NRS $\left(R_{\mathrm{S}}=0.62, P<0.001\right)$ (Table 4$)$.

\section{Discussion}

This study investigated the responsiveness and the minimum important change of the ODI-I on a sample of 151 SPL patients who attended a physical therapy program. The main ODI-I score (22.8) and the main post-treatment changes $(-10.7)$ were similar although slightly lower than 
Table 1 Characteristics of the sample

\begin{tabular}{|c|c|c|c|}
\hline Variable & Category & $N$ & $\%$ \\
\hline \multirow[t]{10}{*}{ Age (years) in classes } & $18-24$ & 19 & 12.7 \\
\hline & $25-29$ & 11 & 7.3 \\
\hline & $30-34$ & 13 & 8.7 \\
\hline & $35-39$ & 16 & 10.7 \\
\hline & $40-44$ & 17 & 11.3 \\
\hline & $45-49$ & 12 & 8.0 \\
\hline & $50-54$ & 15 & 10.0 \\
\hline & $55-59$ & 13 & 8.7 \\
\hline & $60-64$ & 17 & 11.3 \\
\hline & $>65$ & 17 & 11.3 \\
\hline \multirow[t]{2}{*}{ Marital status } & Married & 91 & 60.3 \\
\hline & Unmarried & 60 & 39.7 \\
\hline \multirow[t]{6}{*}{ Work activity } & Student & 16 & 10.6 \\
\hline & Employee & 65 & 43.0 \\
\hline & Self-employed & 26 & 17.2 \\
\hline & Retired & 24 & 15.9 \\
\hline & Houseworker & 19 & 12.6 \\
\hline & Unemployed & 1 & 0.7 \\
\hline \multirow[t]{4}{*}{ Education } & Elementary school & 4 & 2.6 \\
\hline & Mid-school & 13 & 8.6 \\
\hline & Upper school & 74 & 49.0 \\
\hline & University & 60 & 39.7 \\
\hline \multirow[t]{2}{*}{ Smoker } & Yes & 32 & 21.2 \\
\hline & No & 119 & 78.8 \\
\hline \multirow[t]{3}{*}{ Level of spondylolisthesis } & $\mathrm{L} 5 / \mathrm{S} 1$ & 113 & 74.8 \\
\hline & L4/L5 & 36 & 23.8 \\
\hline & $\mathrm{L} 4 / \mathrm{L} 5$ and $\mathrm{L} 5 / \mathrm{S} 1$ & 2 & 1.3 \\
\hline \multirow[t]{2}{*}{ Type of spondylolisthesis } & Isthmic & 102 & 67.5 \\
\hline & Degenerative & 49 & 32.5 \\
\hline \multirow[t]{4}{*}{ Grade of spondylolisthesis } & I & 123 & 81.5 \\
\hline & II & 27 & 17.9 \\
\hline & III & 1 & 0.7 \\
\hline & IV & - & - \\
\hline \multirow[t]{3}{*}{ Pain duration in months } & $0-1$ & 22 & 14.6 \\
\hline & $2-3$ & 22 & 14.6 \\
\hline & $>3$ & 107 & 70.9 \\
\hline \multirow[t]{2}{*}{ Referred pain } & Yes & 74 & 49.3 \\
\hline & No & 76 & 50.7 \\
\hline \multirow[t]{4}{*}{$\operatorname{Drugs}^{\mathrm{a}}$} & Antidepressants & 7 & 4.6 \\
\hline & Analgesics & 29 & 19.2 \\
\hline & NSAIDs $^{\mathrm{b}}$, steroids & 12 & 8 \\
\hline & Muscle relaxants & 1 & 0.7 \\
\hline \multirow[t]{7}{*}{ Comorbidities $^{\mathrm{c}}$} & Heart disease & 4 & 2.6 \\
\hline & Respiratory disease & 2 & 1.3 \\
\hline & Enteric disease & 8 & 5.2 \\
\hline & Endocrinal disease & 2 & 1.3 \\
\hline & Renal disease & 1 & 0.7 \\
\hline & Orthopedic dysfunction & 9 & 5.9 \\
\hline & Anxiety/depression & 9 & 5.9 \\
\hline
\end{tabular}

\footnotetext{
${ }^{a}$ Some patients took more than one drug

${ }^{\mathrm{b}}$ NSAIDs non-steroidal anti-inflammatory drugs

${ }^{c}$ Some patients had more than one comorbidity
}

those calculated by Fairbank and Pynsent (26.6 and 14.4, respectively) in SPL subjects [2]. The best cut-off point $(-7.5)$ was lower than that found in a previous study on ODI-I in non-specific LBP (-9.5) [6], whereas the AUCs are similar (0.76 and 0.71 , respectively), suggesting moderate discriminating ability of this questionnaire.

The changes in ODI-I scores are in line with the values proposed for the minimum important change by Ostelo et al. [4], i.e., a reduction of 10 points, or a decrease of $30 \%$ compared to baseline.

Our results showed a relevant and comparable effect of the treatment on the ODI-I score. The changes in ODI-I score also appeared significantly related to the amount of perceived improvement and were coherent with the changes in the other outcome measures concerning lumbar pain and muscular endurance. The correlation between pain change scores and ODI change scores is in line with a previous study on patients submitted to spinal surgery [21]. Unfortunately, we cannot comment about the correlation with bridge tests due to the lack of published studies on this topic.

Our findings should be analyzed in the light of some factors that can affect the results of outcome measures in LBP. First, we consider that the expectation of improvements and the stage of the pain can influence the rates of change which are higher in acute compared to chronic subjects [22]. Moreover, due to the multifactorial origin of the LBP, we cannot exclude that lumbar disc derangements or other dysfunctions instead of SPL caused pain [24].

Furthermore, both the variability within the population and the inter-individual differences can influence the responsiveness of a measure. As observed by Lauridsen et al. [23], an increase of $25 \%$ in ODI baseline score provokes a 12-point augmentation in the minimum important change of this measure. Demoulin et al. [24] also stressed the relevance of the variability of the time between evaluations on the responsiveness of a measure. In our sample, duration of the pain was variable and questionnaires were administered only at baseline and immediately after the treatment, without any further follow-up. This suggests caution in interpreting the results.

\section{Study limitations}

The limitations of this study can be related to the failure of the patient to assess the change, which could also be reflected in the final disability score. It can cause measurement errors on global evaluation and errors on disability assessment as correlated. Moreover, a one-question global assessment score may not differentiate between quantitative and qualitative perception of change [25].

Other limitations are related to the execution of clinical tests [26], because the same physical therapists performed the clinical tests and conducted the treatments. 
Table 2 Instruments scores before and after treatment

\begin{tabular}{|c|c|c|c|c|}
\hline \multirow[t]{2}{*}{ Method } & \multirow[t]{2}{*}{$N$} & \multicolumn{3}{|c|}{ Mean \pm standard deviations (SD) } \\
\hline & & Pre-treatment & Post-treatment & Difference within groups \\
\hline \multicolumn{5}{|l|}{ NRS } \\
\hline Improved & 120 & $42.6 \pm 21.8$ & $14.5 \pm 16.0$ & $-28.1 \pm 20.4$ \\
\hline Not improved & 31 & $52.4 \pm 20.0$ & $39.5 \pm 19.5$ & $-12.9 \pm 20.0$ \\
\hline Total & 151 & $44.6 \pm 21.7$ & $19.6 \pm 19.5$ & $-25.0 \pm 20.6$ \\
\hline \multicolumn{5}{|l|}{ ODI-I } \\
\hline Improved & 120 & $22.2 \pm 13.1$ & $9.7 \pm 8.5$ & $-12.5 \pm 10.0$ \\
\hline Not improved & 31 & $23.2 \pm 14.5$ & $21.1 \pm 16.3$ & $-2.2 \pm 13.1$ \\
\hline Total & 151 & $22.4 \pm 12.0$ & $12.0 \pm 11.4$ & $-10.4 \pm 10.4$ \\
\hline \multicolumn{5}{|l|}{ PBT } \\
\hline Improved & 120 & $21.7 \pm 25.2$ & $43.0 \pm 32.8$ & $21.2 \pm 23.4$ \\
\hline Not improved & 31 & $14.4 \pm 20.0$ & $20.0 \pm 23.8$ & $5.6 \pm 9.6$ \\
\hline Total & 151 & $20.2 \pm 24.7$ & $38.3 \pm 32.4$ & $18.0 \pm 19.6$ \\
\hline \multicolumn{5}{|l|}{ SBT } \\
\hline Improved & 120 & $76.8 \pm 60.2$ & $125.4 \pm 54.8$ & $48.6 \pm 50.6$ \\
\hline Not improved & 31 & $56.3 \pm 54.8$ & $70.7 \pm 59.0$ & $14.5 \pm 27.5$ \\
\hline Total & 151 & $72.6 \pm 59.6$ & $114.2 \pm 59.7$ & $41.6 \pm 50.0$ \\
\hline
\end{tabular}

Data are expressed as means \pm standard deviations (SD)

$N$ number, $O D I-I$ Oswestry Disability Index (Italian version), NRS numerical rating scale, $P B T$ prone bridge test, $S B T$ supine bridge test

Table 3 Responsiveness of NRS and ODI-I

\begin{tabular}{|c|c|c|c|}
\hline Method & $\begin{array}{l}\text { Value } \\
\text { Total }\end{array}$ & Improved & Not improved \\
\hline \multicolumn{4}{|l|}{ NRS } \\
\hline Minimum detectable change & 9.77 & 7.98 & 9.75 \\
\hline Effect size & 1.15 & 1.29 & 0.65 \\
\hline Effect size (Guyatt) & 1.25 & 1.41 & 0.65 \\
\hline Standardized response mean & 1.21 & 1.38 & 0.65 \\
\hline Optimal cut-off point (AUC, sensitivity, specificity) & $17.5(0.85,90.3,37.5)$ & & \\
\hline \multicolumn{4}{|l|}{ ODI-I } \\
\hline Minimum detectable change & 5.72 & 4.23 & 8.14 \\
\hline Effect size & 1.0 & 0.95 & 0.15 \\
\hline Effect size (Guyatt) & 0.87 & 0.86 & 0.15 \\
\hline Standardized response mean & 1.0 & 1.25 & 0.17 \\
\hline Optimal cut-off point (AUC, sensitivity, specificity) & $7.5(0.76,90.3,56.7)$ & & \\
\hline
\end{tabular}

ODI-I Oswestry Disability Index (Italian version), NRS numerical rating scale

As most of the patients reported a better condition on GPE, we cannot comment about the responsiveness of the ODI-I in subjects who reported a worsened state.

The results of this study cannot be applied to different categories of specific LBP, because our inclusion criteria only selected subjects with lumbar SPL.

This series included only patients treated non-operatively (as definitive treatment or as an initial attempt before proceeding with an indication of surgical treatment). Thus, our findings could not be applicable to those patients who having more severe forms of spondylolisthesis needed surgical treatment first. Finally, as our sample included both isthmic and degenerative spondylolisthesis, we cannot draw any conclusion about a difference in responsiveness between these two groups.

Suggestions for future studies are to investigate the responsiveness of the ODI-I in various LBP subgroups, submitted to different treatments or assessed with other outcome measures. 
Table 4 Spearman's rank correlation coefficients between the ODI-I and other parameters

\begin{tabular}{lcl}
\hline Basal metabolic data & Spearman's $r$ & $P$ value \\
\hline Age & 0.21 & 0.001 \\
NRS & 0.62 & 0.001 \\
GPE & 0.36 & 0.001 \\
PBT & -0.45 & 0.001 \\
SBT & -0.48 & 0.00 \\
\hline
\end{tabular}

$N R S$ numerical rating scale, GPE global perceived effect, $P B T$ prone bridge test, $S B T$ supine bridge test

In conclusion, this study demonstrated a moderate responsiveness of the ODI-I in detecting clinical changes after physical therapy treatment in subjects with symptomatic specific LBP associated to lumbar SPL. These findings are coherent with those published in the literature with different LBP samples.

\section{Compliance with ethical standards}

Conflict of interest The authors did not declare any conflict of interest.

Patient consent All patients gave informed consent prior to inclusion in the study.

Ethical Approval All procedures involving human participants were in accordance with the 1964 Helsinki declaration and its later amendments. The study was approved by the Institutional Review Board.

Funding No funding was delivered for this research.

Open Access This article is distributed under the terms of the Creative Commons Attribution 4.0 International License (http://creative commons.org/licenses/by/4.0/), which permits unrestricted use, distribution, and reproduction in any medium, provided you give appropriate credit to the original author(s) and the source, provide a link to the Creative Commons license, and indicate if changes were made.

\section{References}

1. de Vet HC et al (2006) Minimal changes in health status questionnaires: distinction between minimally detectable change and minimally important change. Health Qual Life Outcomes 4:54

2. Fairbank JC, Pynsent PB (2000) The Oswestry Disability Index. Spine 22:2940-2952 (discussion 2952)

3. Cleland J et al (2011) Assessing dimensionality and responsiveness of outcomes measures for patients with low back pain. Pain Pract 11:57-69

4. Ostelo RW et al (2008) Interpreting change scores for pain and functional status in low back pain: towards international consensus regarding minimal important change. Spine 33:90-94

5. Monticone M et al (2009) Development of the Italian version of the Oswestry Disability Index (ODI-I): a cross-cultural adaptation, reliability, and validity study. Spine 34:2090-2095

6. Monticone M et al (2012) Responsiveness of the Oswestry Disability Index and the Roland Morris Disability Questionnaire in
Italian subjects with sub-acute and chronic low back pain. Eur Spine J 21:122-129

7. Soini J et al (1993) Spondylodesis augmented by transpedicular fixation in the treatment of olisthetic and degenerative conditions of the lumbar spine. Clin Orthop Relat Res 297:111-116

8. Soini J et al (1993) External transpedicular fixation test of the lumbar spine correlates with the outcome of subsequent lumbar fusion. Clin Orthop Relat Res 293:89-96

9. Tiusanen $\mathrm{H}$ et al (1996) Results of a trial of anterior or circumferential lumbar fusion in the treatment of severe isthmic spondylolisthesis in young patients. J Pediatr Orthop B 5:190-194

10. Soini JR et al (1994) External fixation test in low back pain. Function analyzed in 25 patients. Acta Orthop Scand 65:87-90

11. O'Sullivan PB et al (1998) Altered abdominal muscle recruitment in patients with chronic back pain following a specific exercise intervention. J Orthop Sports Phys Ther 27:114-124

12. Nava-Bringas TI et al (2014) Effects of a stabilization exercise program in functionality and pain in patients with degenerative spondylolisthesis. J Back Musculoskelet Rehabil 27:41-46

13. von Elm E et al (2008) The strengthening the reporting of observational studies in epidemiology (STROBE) statement: guidelines for reporting observational studies. J Clin Epidemiol 61:344-349

14. Tamburrini O, Dalla Palma F (2008) The Radiological Medical Act approved by the SIRM Executive Committee on July 2, 2007. Radiol Med (Torino) 113:319-328

15. Marchetti P, Bartolozzi P (1997) Classification of spondylolisthesis as a guideline for treatment. 2nd ed. Lippincott-Raven, Philadelphia, pp 1211-1254

16. Hjermstad MJ et al (2011) Studies comparing numerical rating scales, verbal rating scales, and visual analogue scales for assessment of pain intensity in adults: a systematic literature review. J Pain Symptom Manage 41:1073-1093

17. Schellenberg KL et al (2007) A clinical tool for office assessment of lumbar spine stabilization endurance: prone and supine bridge maneuvers. Am J Phys Med Rehabil 86:380-386

18. Richardson C, Jull G, Hodges, PW, Hides JA (1999) Therapeutic exercise for spinal segmental stabilization in low back pain: scientific basic and clinical approach. $1^{\text {st }}$ edn, Sydney: Churchill Livingstone

19. Hosseinifar $M$ et al (2013) The effects of stabilization and McKenzie exercises on transverse abdominis and multifidus muscle thickness, pain, and disability: a randomized controlled trial in nonspecific chronic low back pain. J Phys Ther Sci 25:1541-1545

20. Guyatt $\mathrm{G}$ et al (1987) Measuring change over time: assessing the usefulness of evaluative instruments. J Chronic Dis 40:171-178

21. DeVine $J$ et al (2011) Evaluating the correlation and responsiveness of patient-reported pain with function and quality-of-life outcomes after spine surgery. Spine 36:S69-74

22. Grotle $\mathrm{M}$ et al (2004) Concurrent comparison of responsiveness in pain and functional status measurements used for patients with low back pain. Spine 29:E492-501

23. Lauridsen $\mathrm{HH}$ et al (2006) Responsiveness and minimal clinically important difference for pain and disability instruments in low back pain patients. BMC Musculoskeletal Disord 7:82

24. Demoulin C et al (2010) What factors influence the measurement properties of the Roland-Morris disability questionnaire? Eur $\mathrm{J}$ Pain 14:200-206

25. Terwee CB et al (2010) Mind the MIC: large variation among populations and methods. J Clin Epidemiol 63:524-534

26. Carlsson H, Rasmussen-Barr E (2013) Clinical screening tests for assessing movement control in non-specific low-back pain. A systematic review of intra- and inter-observer reliability studies. Man Ther 18:103-110 